\title{
LNCRNA expression landscape and specificity between brain regions
} Adewale Joseph, Ogunleye ${ }^{1}$, Umair Ali ${ }^{1}$, Michael Juwon, Olufemi ${ }^{2}$

1. Department of Biological and Medical Physics, Moscow Institute of Physics and Technology, 141701 Dolgoprudny, Russia

2. Genomic Research in Biomedicine Laboratory, Nigerian Institute of Medical Research, Lagos, Nigeria

Adewale Joseph, Ogunleye, Graduate Student, (Group of Regulatory Transcriptomics and Epigenomics, Institute of Bioengineering, Research Center of Biotechnology, Russian Academy of Science, 117312 Moscow, Russia)

(ogunleie.ad@phystech.edu)

Keywords: IncRNA, Brain, transcription, splicing, tissue differentiation

\author{
Abbreviations: \\ IncRNA (Long non coding Ribonucleic acid) \\ RNA (Ribonucleic acid) \\ DNA (Deoxyribonucliec acid) \\ mRNA (Messenger Ribonucleic acid) \\ GTEx (Genotype-Tissue Expression ) \\ MAF (Minor allele frequency) \\ sQTL (Splicing quantitative trait loci) \\ PAGA (Partition-based graph abstraction)
}




\begin{abstract}
:
Long noncoding RNAs (IncRNAs) are transcribed into low potential protein coding RNA molecules, which account for over $70 \%$ of mammalian transcriptional products. The role of IncRNAs and their expression is still largely unknown, and the subject of recent investigations. Here, we used bulk RNA sequencing data from the Genotype-Tissue Expression (GTEx) project to reveal the occurrence and identify the specificity of IncRNAs in 13 brain regions (1000 samples). We observed that these highly specific IncRNA were co-expressed with previously known mRNA markers for the 13 study regions of the brain. Further investigation revealed that splicing could influence the divergent biogenesis and enrichment of specific IncRNA alleles in different brain regions. Overall, we demonstrate the use of IncRNA as an independent tool for deconvolving brain regions and further highlights its use for cell-type identification from bulk transcriptome data.
\end{abstract}

\title{
Introduction:
}

LncRNA are transcriptional products with $>200$ nucleotides without a distinct open reading frame and poor protein coding potential [1,2]. LncRNA can regulate major cellular processes such as transcription and epigenetics by initiating signal transduction [3,4], sponging microRNA [5-7], acting as a scaffold for assembly molecules and guiding proteins to their targets $[1,8,9]$. Unlike messenger RNA (mRNA), IncRNA are localized in the nucleus where they directly interact with the genome to influence overall cell development $[2,10]$. It is believed that IncRNAs are involved in modulating gene expression, and their canonical functions start at a genetic locus. However, IncRNAs are transcribed from enhancers,called eRNAs.both are heterogenous molecules and different from each other but perform the same function in the progress of mRNA transcription and translation [11-14]. However IncRNAs do not possess protein coding ability, spatiotemporal-specific expression patterns have enhanced the different functions and complex processes of IncRNAs [15]. For the functionality of IncRNAs the precise processing, tissue specificity, subcellular localization and differential expression of IncRNAs can be used as arguments. It could be taken logically as that these observations are also 
consistent with IncRNAs being the product of noisy transcription. LncRNAs exhibit tissue-type, cell-type and cell-type condition specificity in their expression pattern. Generally, about $11 \%$ of IncRNAs are ubiquitous in all human tissues [16]. LncRNAs are abundantly expressed in the brain, however, the tissues have been shown to be non-overlapping in their expression patterns $[17,18]$. For example, high throughput RNA in-situ hybridization analysis of the Allen Brain Atlas (adult mouse brain) $[19,20]$ identified 849 out of 1328 IncRNAs to be associated with a distinct brain region, cell or subcellular compartment [21]. Due to the apparent profile of distinct IncRNA expression, brain regions such as hippocampus, cerebral cortex, olfactory bulb and cerebellum, some studies have proposed IncRNAs as biomarkers for some brain regions [17,18,22]. It is also safe to assume that this distinct specificity within regions suggests that IncRNA may play a role in the development and homeostasis of brain regions as opposed to the popular notion that they are transcriptional noise. Resolving the population and abundance of IncRNA within brain regions poses several fundamental constraints. The poor inter-species conservation of IncRNA raises evolutionary appreciation but may contradict extrapolation of functional insights. Furthermore, IncRNAs show transient expression between developmental stages $[18,23]$ which may nullify the significance of a comprehensive catalogue. In this study, we attempted to resolve the distinct population of IncRNAs between 13 specific regions in 1000 adult human brains RNA samples as documented by the GTEx consortium [22,24,25]. The enrichment of different IncRNAs within variable brain regions was apparent. Since the GTEx data also provides a significant resource on splicing, we investigated possible contributions of the special splicing program of the brain to IncRNA expression.

\section{Methods}

\section{Bulk RNA-Seq data preprocessing}

Bulk RNA-Seq data (measured in transcript per million; TPM) was retrieved from the Genotype Tissue Expression (GTEx) consortium (https://gtexportal.org/home/datasets) [22,24,25]. For the purpose of this study, we randomly selected 1000 brain samples containing 13 brain regions (cortex, frontal cortex, nucleus accumbens, caudate, cerebellar hemisphere, cerebellum, putamen, hypothalamus, substantia nigra, hippocampus, spinal cord, anterior singlet cortex and amygdala) as classified GTEx. 5885 IncRNAs were selected based on the annotations in GENCODE release $32[26,27]$. Due to the unstable nature of IncRNA nomenclature, we decided to stick with the much more ensembl ID.

\section{Cluster analysis}

We performed semi-supervised clustering to group IncRNAs into distinct expression clusters using Uniform Manifold Approximation and Projection and manual cluster labelling. First, we removed IncRNAs with non-variable expressions, and performed tissue-wise count normalization. We implemented UMAP within ScanPy [28] for 1754 IncRNAs and recovered 13 biologically relevant clusters with non-repetitive transcript profiles. Cluster analysis presents a unique challenge associated with defining the biological identity of each cluster. This was solved by ranking IncRNAs within each cluster using Wilcoxon's rank sum analysis after which we assigned labels manually using GTEx transcript browser.

\section{Splicing across regions}


Generally, IncRNAs are weakly spliced, but such rare events are known to contribute to vary within tissues [29,30]. Splicing quantitative trait loci (sQTL) (minor allele frequency) data was retrieved from GTEx and preprocessed for the IncRNAs expressed in the 13 brain regions. To estimate the variation of splicing between regions, we performed two independent analyses; (i) We inferred IncRNA trajectories between the regions and the trajectory pseudotime using partition-based graph abstraction (PAGA). (ii) We performed hierarchical clustering for each region based on the presence and abundance of IncRNA splicing alleles and ranked the outputs. The ranked output of each of these independent analyses was manually compared.

\section{Cerebellar cell-type identification}

Upon identification of the highly variable IncRNAs in each region, we performed pairwise correlation analysis to determine co-expression pattern between these IncRNAs and 4 highly expressed mRNAs markers in each of the 4 cerebellar cells (Granule-cell, Purkinje-cell, Astrocyte, Oligodendrocyte) identified by colorimetric in-situ hybridization experiments from the Allen brain atlas (ABA) [20,31]. ABA provides at least 5 cell-specific mRNA markers for each cell; granule cell (Zranb2, Capn7, Mfap3, Ubr1 and Tiam1), purkinje cell (Car8, Large, Pvalb, Gsbs and Lmo7), astrocyte (Dao1, Tmem47, Mlc1, Sox21 and 120009022Rik) and oligodendrocyte (Hspa2, Anln, Mobp, Mylk and Klk6) [31]. We collected count data for all 20 mRNA from the same GTEx dataset we are using. Upon pairwise correlation, we used k-mean clustering to iteratively identify the maximum number of biologically relevant clusters that can be extracted from the correlation matrix. Pairwise correlation was performed with pandas cor $r$ function, k-mean clustering and visualization was done in ggplot2 (R).

Figure 1

\section{Results and Discussion}

There is a gradual transition in the population IncRNAs in anatomically close brain regions Characterizing tissue-specific expression of IncRNA is critical to understanding their phenotypic consequences. In the current study, we deconvolved 13 brain regions using bulk IncRNA expression data from 1000 de-identified brain regions to understand the relationship between IncRNA and observed phenotypes. Upon data preprocessing, we focused on 1750 out of 5885 IncRNAs with hypervariable expression profiles to describe region-specific transcription profiles. Using Uniform Manifold Approximation and Projection (UMAP) embedding, we identified 13 clusters based on IncRNA expression count for 1000 brain regions (Figure 2a). To confirm that these clusters are biological correspondents of the brain regions, we ranked all IncRNAs in the cluster using p-value estimates from Wilcoxon sum rank analysis (Supplementary Table 1). We then manually queried the GTEx transcript browser using the top 5 ranked IncRNA in each cluster to reflect the true biological identity.

We were able to detect a small population of IncRNA (usually 3-5) that are really specific to each region. We further observed a gradual but discrete shift in the presence and abundance of IncRNAs between the different brain regions. This is particularly obvious for regions that are anatomically close such as cerebellum and cerebellar hemisphere, cortex and frontal cortex and brain regions in the basal ganglia (substantia nigra, hypothalamus and putamen). This might suggest a spatial link in the abundance and gradual spread of IncRNA over closely associated regions of the brain (Figure $2 b-d$ ). In a recent preprint performed by [32], an 
unsupervised hierarchical clustering to segregate the brain regions based on the transcriptome of the chimpanzee's brain. In an attempt to correlate the transcriptome transitions with evolutionary development of brain regions, they recovered putamen, substantia nigra and hypothalamus in this order. The ability of IncRNA to also provide a similar transcriptome transition between the regions suggests a role for IncRNA in their differentiation.

\section{Figure 2}

Also, some ubiquitously expressed IncRNAs such as MEG3, OBSCN, ZFAS1, SNHG15 and PART1 were found as part of the top ranked genes in many clusters. Previous studies have shown that ubiquitous IncRNAs are important for basal cell function and some house-keeping roles [33]. We suggest that they may cooperate with the singly expressed IncRNAs to stabilize the core IncRNA network that defines each brain region.

\section{Splicing}

LncRNAs are known to be rarely spliced due to their weak internal splicing signals which is a consequence of the relatively longer distance between the 5' splice site, 3' splice sites and branch point $[22,29]$. Nevertheless, a few IncRNA splicing variants have been identified and they correlate with tissue variation. Indeed, we retrieved 109 IncRNAs with splice allele variants (minor alleles) across all brain regions from the GTEx database which agrees with the low splicing frequency of IncRNAs. We created a minor allele frequency (MAF) matrix between these 109 IncRNA and the 13 brain regions. To capture the influence of IncRNA splicing, 2 independent strategies were performed using the MAF matrix. First, we selected the cerebellum as an initial root to infer a developmental trajectory and prediction of the pseudo-temporal transition IncRNAs between the brain regions based on the 1750 hypervariable IncRNAs. Secondly, we performed ranked hierarchical clustering of the MAF matrix (109 IncRNAs). We then manually compared the two ranked outputs in a pairwise matrix. Although we could not infer a direct cause-effect relationship between splicing abundance and tissue variation, our analysis showed that brain regions with low splicing allele frequencies (substantia nigra, frontal cortex, amygdala, spinal cord and putamen) were terminal endpoints of the trajectory analysis and hierarchical tree (figure 3a-c). Implicitly, it can be assumed that the trajectories represent the differentiation of brain regions on a time scale, as well as a qualitative and quantitative transition in the estimate of IncRNAs splicing across different brain regions.

\section{Figure 3}

\section{Cerebellar cell-type identification via mRNA-IncRNA co-expression analysis}

The cerebellum regions were richly represented in the GTEx dataset, with distinct IncRNA markers. Using pairwise correlation analysis, we identified co-expression patterns between mRNA markers (retrieved from ABA, see methods) and total IncRNA transcriptome of the cerebellum. We further dissected the correlation matrix into 4 clusters (k-mer, see methods) that matched each cell type (figure 4). The identity of the clusters were verified by the mRNA marker in each cluster. Overall, we identified IncRNA markers for granule cells (IncRNA: RP11-350N15.4-001, RP3-405J10.3-001), purkinje cells (IncRNA: LINC00304-001, 
LINC00304-002, RP1-310013.7-001, RP13-895J2.2, RP13-895J2.2-002), astrocytes (IncRNA: AC005786.7, RP11-23P13.6-001, RP11-23P13.6-002) and oligodendrocytes (IncRNA: MEG3-026, MEG3-004,) (see supplementary table 2).

\section{Figure 4}

Of important note is the expression and specificity of MEG3 and its variants to cerebellar oligodendrocytes. Previous studies have noted the absence of MEG3 in the nucleus of mature oligodendrocytes. However, a recent study performed by [34], identified 3 distinct populations of oligodendrocyte progenitor cells (OPCs; OPC1, OPC2 and OPC3) with MEG3 expression in only OPC3. Our results provide supporting evidence and suggest that MEG3 expression may be crucial to OPC lineage migration into the cerebellum. The functional significance of LINC00304 and its variants is currently not well understood. Nevertheless, we detected its high expression in the cerebellar cortex of the developing human brain atlas as curated by the harmonizome database [35], while [36] profiled it as a regulator of CpG specific DNA methylation in obesity patients. Unfortunately we could not profile specific roles for astrocyte and granule cell-specific IncRNAs. Overall, we reflect the essentiality of IncRNAs as a sufficient molecular tool for deconvolving the cell types even from bulk IncRNA data.

\section{Conclusion}

Overall, we investigated the expression of IncRNA with respect to their regional specificity in the brain. We showed that some subset of IncRNA could efficiently form a network that can define the identity of each brain region. We further leveraged on the cell specificity of IncRNAs to predict four cell types in the cerebellum. We propose that future studies should verify the functional modularity of these IncRNAs to cell physiology using targeted approaches.

\section{Conflicts of interest}

None to be declared.

\section{Images and Captions:}

\section{Figure 1:}




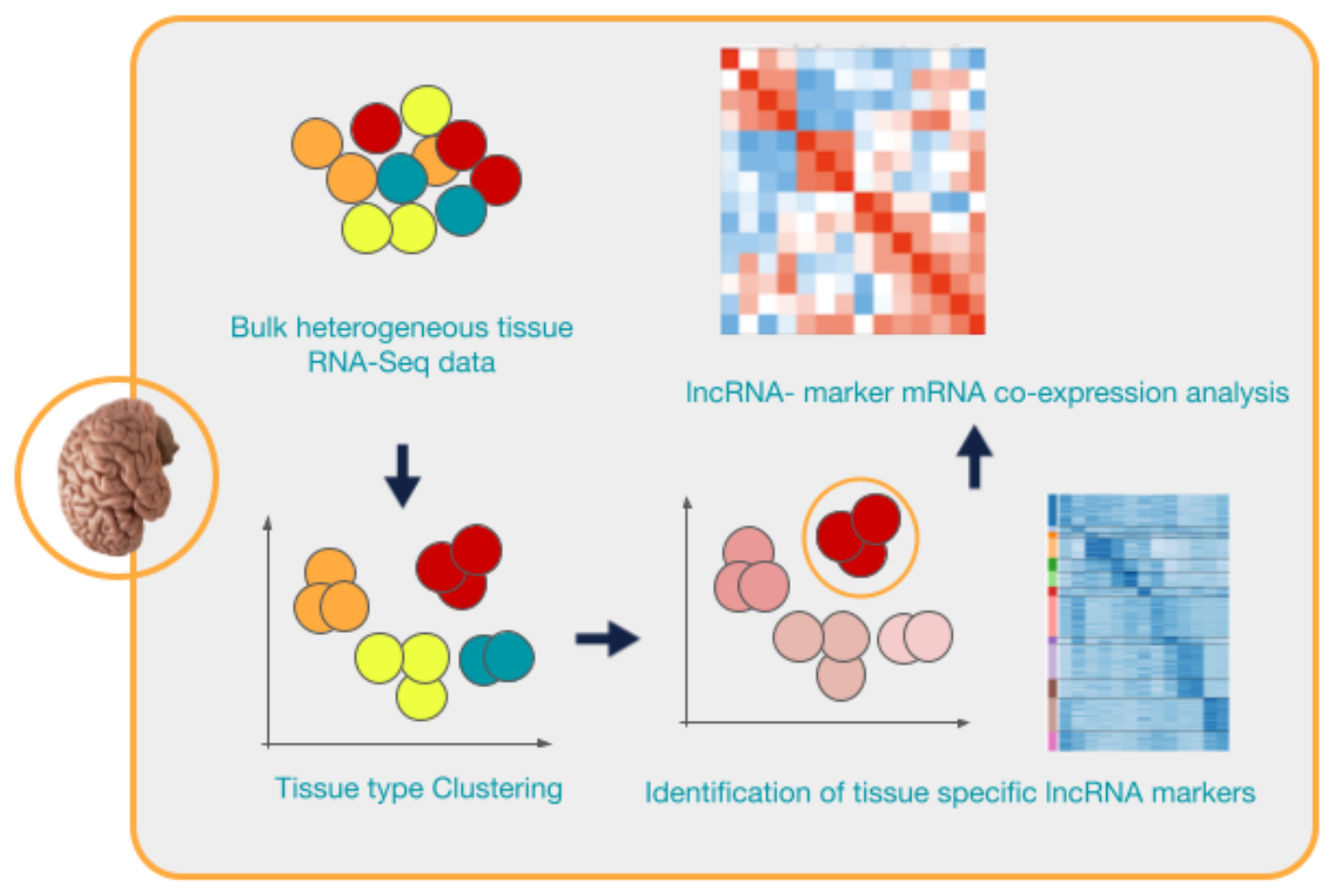

Figure 1: Workflow for the methods used in this study. We collected data from the GTEx dataset, performed UMAP clustering, manually identified the biological identity of the clusters and finally, used co-expression correlation to identify four cell types in the cerebellum. 
a

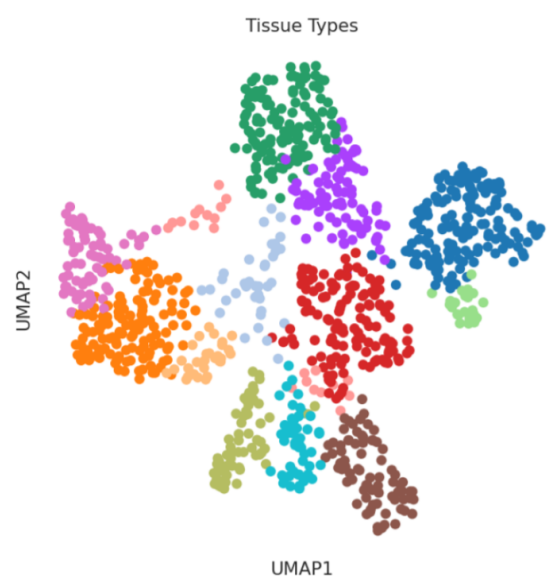

- Anterior cingulate cortex (BA24)

- Spinal cord (cervical c-1)

- Hippocampus

- Substantia nigra

- Hypothalamus

- Putamen

- Cerebellum

- Caudate (basal ganglia)

- Nucleus accumbens (basal ganglia)

- Frontal Cortex (BA9)

- Cortex
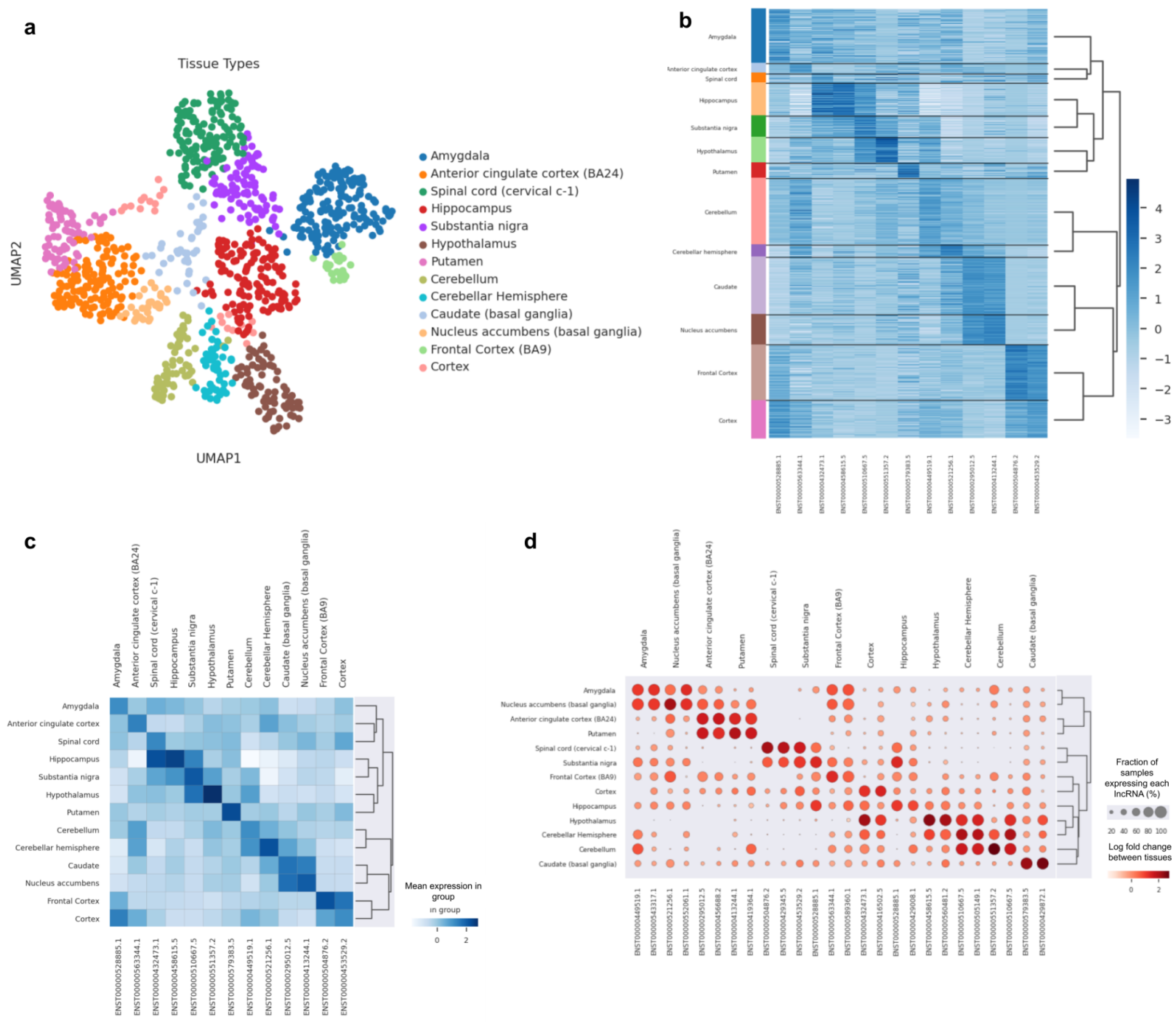

d
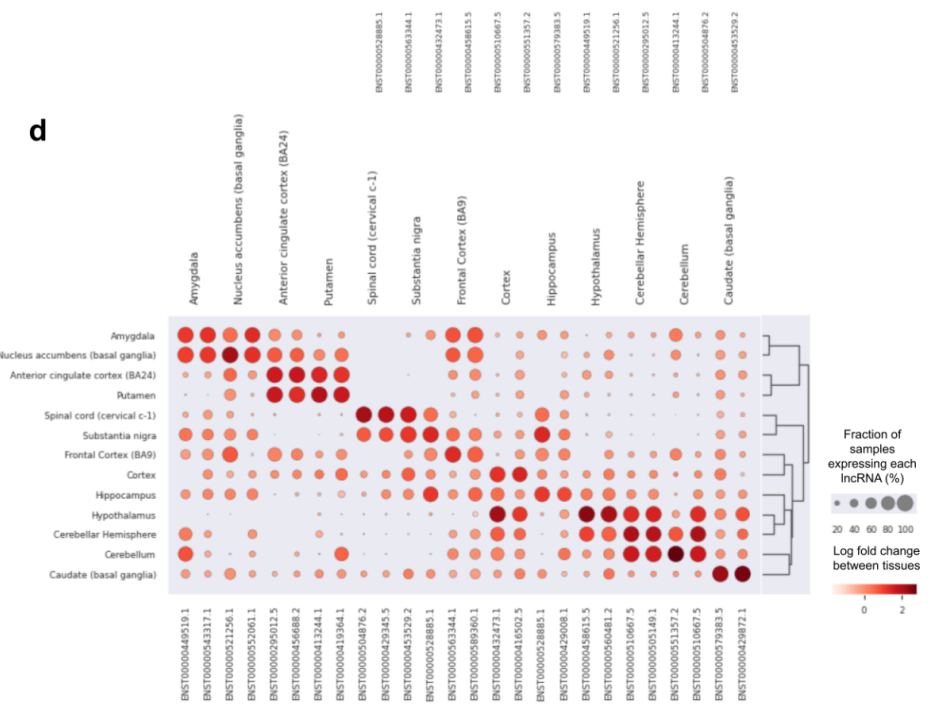

Figure 2: (a) Clustering and identification of the 13 brain regions from UMAP calculations from 1000 de-identified samples and 1750 IncRNAs. (b) Heatmap showing the enrichment in expression of each brain region marker based on Log-transformed normalized expression. (c) Mean expression of each IncRNA marker compared over all the brain region clusters. (d) Log2 fold change of the top 2 IncRNA markers between the different brain regions and the percentage of the number of samples expressing each IncRNA marker in each group.

(NB: Due to the unstable nature of IncRNA nomenclature, we decided to stick with the much more ensembl ID). 

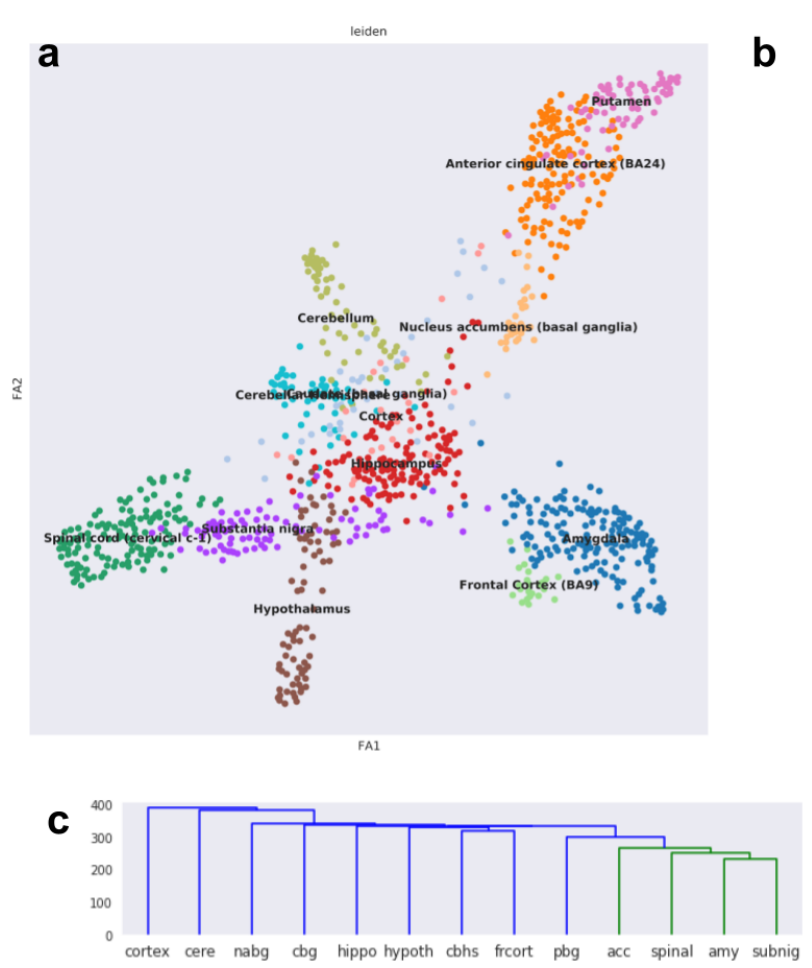

b

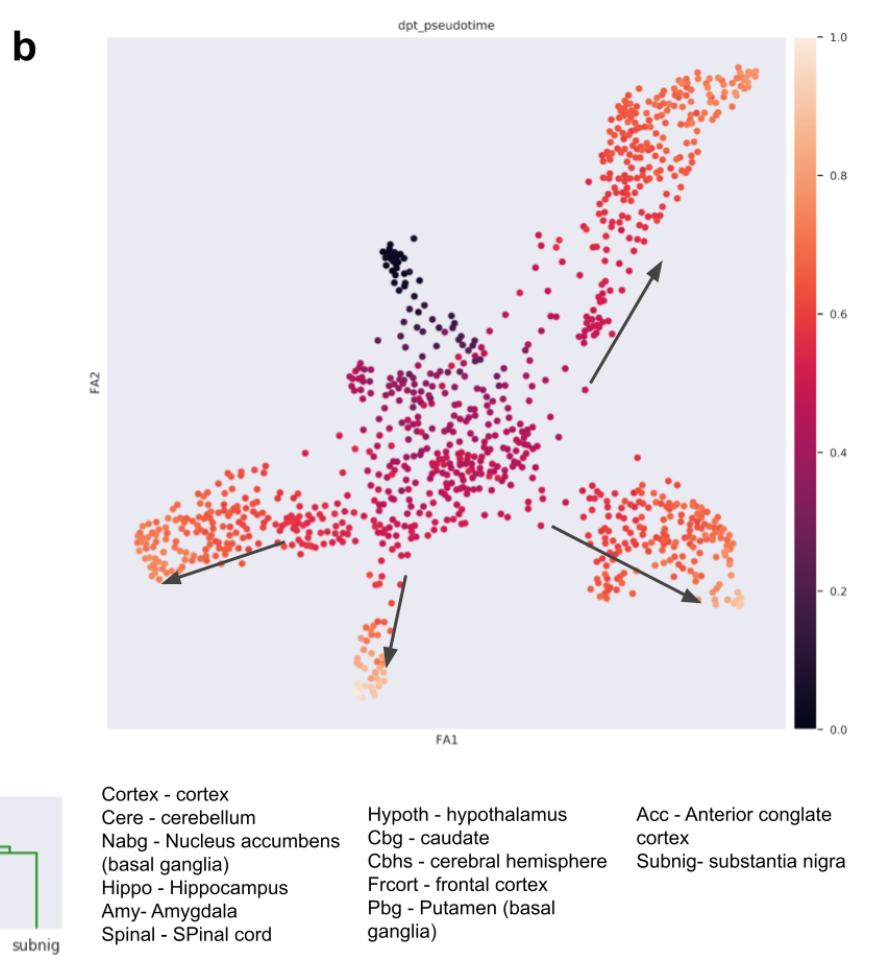

Figure 3: (a) trajectory inference reflects the pseudo-temporal transition and connectivity of IncRNAs between brain regions (b) Trajectory pseudotime analysis (with cerebellum as a starting point) shows that substantia nigra, frontal cortex, amygdala, spinal cord and putamen as endpoints of the trajectory. (c) Anterior cingulate cortex, substantia nigra, amygdala and spinal cord are terminal endpoints of the hierarchical clustering. Compared with the observation of the trajectory analysis, they are terminal endpoints of the pseudotime prediction. 

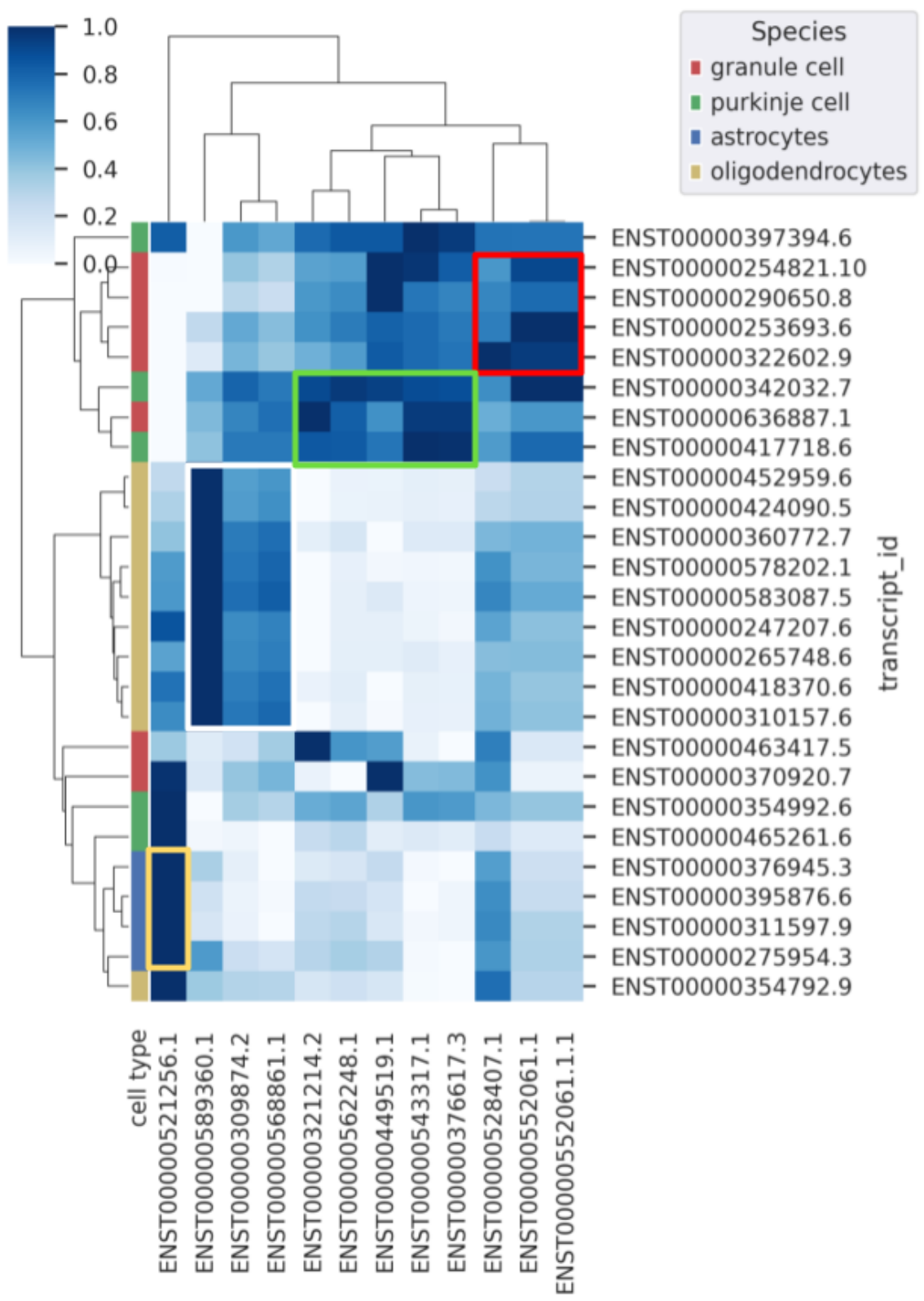

Figure 4: Co-expression analysis of the IncRNA and marker mRNAs predicts four cerebellar cell-types; granule cells (red box), purkinje cells (green box), astrocytes (white box) and oligodendrocytes (yellow box). 


\section{References:}

[1] K.C. Wang, H.Y. Chang, Molecular mechanisms of long noncoding RNAs, Mol. Cell. 43 (2011) 904-914.

[2] R.-W. Yao, Y. Wang, L.-L. Chen, Cellular functions of long noncoding RNAs, Nat. Cell Biol. 21 (2019) 542-551.

[3] A. Lin, C. Li, Z. Xing, Q. Hu, K. Liang, L. Han, C. Wang, D.H. Hawke, S. Wang, Y. Zhang, Y. Wei, G. Ma, P.K. Park, J. Zhou, Y. Zhou, Z. Hu, Y. Zhou, J.R. Marks, H. Liang, M.-C. Hung, C. Lin, L. Yang, The LINK-A IncRNA activates normoxic HIF1 $\alpha$ signalling in triple-negative breast cancer, Nat. Cell Biol. 18 (2016) 213-224.

[4] A. Lin, Q. Hu, C. Li, Z. Xing, G. Ma, C. Wang, J. Li, Y. Ye, J. Yao, K. Liang, S. Wang, P.K. Park, J.R. Marks, Y. Zhou, J. Zhou, M.-C. Hung, H. Liang, Z. Hu, H. Shen, D.H. Hawke, L. Han, Y. Zhou, C. Lin, L. Yang, The LINK-A IncRNA interacts with Ptdlns $(3,4,5) P 3$ to hyperactivate AKT and confer resistance to AKT inhibitors, Nat. Cell Biol. 19 (2017) 238-251.

[5] M.D. Paraskevopoulou, A.G. Hatzigeorgiou, Analyzing MiRNA-LncRNA Interactions, Methods Mol. Biol. 1402 (2016) 271-286.

[6] X.-S. Wu, F. Wang, H.-F. Li, Y.-P. Hu, L. Jiang, F. Zhang, M.-L. Li, X.-A. Wang, Y.-P. Jin, Y.-J. Zhang, W. Lu, W.-G. Wu, Y.-J. Shu, H. Weng, Y. Cao, R.-F. Bao, H.-B. Liang, Z. Wang, Y.-C. Zhang, W. Gong, L. Zheng, S.-H. Sun, Y.-B. Liu, LncRNA-PAGBC acts as a microRNA sponge and promotes gallbladder tumorigenesis, EMBO Rep. 18 (2017) 1837-1853.

[7] Y. Yu, F. Gao, Q. He, G. Li, G. Ding, IncRNA UCA1 Functions as a ceRNA to Promote Prostate Cancer Progression via Sponging miR143, Mol. Ther. Nucleic Acids. 19 (2020) 751-758.

[8] S. Kaneko, R. Bonasio, R. Saldaña-Meyer, T. Yoshida, J. Son, K. Nishino, A. Umezawa, D. Reinberg, Interactions between JARID2 and noncoding RNAs regulate PRC2 recruitment to chromatin, Mol. Cell. 53 (2014) 290-300.

[9] L. Wu, P. Murat, D. Matak-Vinkovic, A. Murrell, S. Balasubramanian, Binding interactions between long noncoding RNA HOTAIR and PRC2 proteins, Biochemistry. 52 (2013) 9519-9527.

[10] R.M. Shallis, R. Wang, A. Davidoff, X. Ma, A.M. Zeidan, Epidemiology of acute myeloid leukemia: Recent progress and enduring challenges, Blood Rev. 36 (2019) 70-87.

[11] T.-K. Kim, M. Hemberg, J.M. Gray, Enhancer RNAs: a class of long noncoding RNAs synthesized at enhancers, Cold Spring Harb. Perspect. Biol. 7 (2015) a018622.

[12] J.-Y. Joo, K. Schaukowitch, L. Farbiak, G. Kilaru, T.-K. Kim, Stimulus-specific combinatorial functionality of neuronal c-fos enhancers, Nat. Neurosci. 19 (2016) 75-83.

[13] K. Schaukowitch, J.-Y. Joo, X. Liu, J.K. Watts, C. Martinez, T.-K. Kim, Enhancer RNA facilitates NELF release from immediate early genes, Mol. Cell. 56 (2014) 29-42.

[14] T.-K. Kim, R. Shiekhattar, Architectural and Functional Commonalities between Enhancers and Promoters, Cell. 162 (2015) 948-959.

[15] H. Cao, C. Wahlestedt, P. Kapranov, Strategies to Annotate and Characterize Long Noncoding RNAs: Advantages and Pitfalls, Trends Genet. 34 (2018) 704-721.

[16] A. Bonetti, F. Agostini, A.M. Suzuki, K. Hashimoto, G. Pascarella, J. Gimenez, L. Roos, A.J. Nash, M. Ghilotti, C.J.F. Cameron, M. Valentine, Y.A. Medvedeva, S. Noguchi, E. Agirre, K. Kashi, Samudyata, J. Luginbühl, R. Cazzoli, S. Agrawal, N.M. Luscombe, M. Blanchette, T. Kasukawa, M. de Hoon, E. Arner, B. Lenhard, C. Plessy, G. Castelo-Branco, V. Orlando, P. Carninci, RADICL-seq identifies general and cell type-specific principles of genome-wide RNA-chromatin interactions, Nat. Commun. 11 (2020) 1018.

[17] B. Yang, Z.-A. Xia, B. Zhong, X. Xiong, C. Sheng, Y. Wang, W. Gong, Y. Cao, Z. Wang, W. 
Peng, Distinct Hippocampal Expression Profiles of Long Non-coding RNAs in an Alzheimer's Disease Model, Mol. Neurobiol. 54 (2017) 4833-4846.

[18] D. He, J. Wang, Y. Lu, Y. Deng, C. Zhao, L. Xu, Y. Chen, Y.-C. Hu, W. Zhou, Q.R. Lu, IncRNA Functional Networks in Oligodendrocytes Reveal Stage-Specific Myelination Control by an IncOL1/Suz12 Complex in the CNS, Neuron. 93 (2017) 362-378.

[19] C. Dang, A. Sodt, C. Lau, B. Youngstrom, L. Ng, L. Kuan, S. Pathak, A. Jones, M. Hawrylycz, The Allen Brain Atlas: Delivering Neuroscience to the Web on a Genome Wide Scale, in: Data Integration in the Life Sciences, Springer Berlin Heidelberg, 2007: pp. 17-26.

[20] S.M. Sunkin, L. Ng, C. Lau, T. Dolbeare, T.L. Gilbert, C.L. Thompson, M. Hawrylycz, C. Dang, Allen Brain Atlas: an integrated spatio-temporal portal for exploring the central nervous system, Nucleic Acids Res. 41 (2013) D996-D1008.

[21] T.R. Mercer, M.E. Dinger, S.M. Sunkin, M.F. Mehler, J.S. Mattick, Specific expression of long noncoding RNAs in the mouse brain, Proc. Natl. Acad. Sci. U. S. A. 105 (2008) 716-721.

[22] A. Saha, Y. Kim, A.D.H. Gewirtz, B. Jo, C. Gao, I.C. McDowell, GTEx Consortium, B.E. Engelhardt, A. Battle, Co-expression networks reveal the tissue-specific regulation of transcription and splicing, Genome Res. 27 (2017) 1843-1858.

[23] Y. Zhao, H. Liu, Q. Zhang, Y. Zhang, The functions of long non-coding RNAs in neural stem cell proliferation and differentiation, Cell Biosci. 10 (2020) 74.

[24] M. Melé, P.G. Ferreira, F. Reverter, D.S. DeLuca, J. Monlong, M. Sammeth, T.R. Young, J.M. Goldmann, D.D. Pervouchine, T.J. Sullivan, R. Johnson, A.V. Segrè, S. Djebali, A. Niarchou, GTEx Consortium, F.A. Wright, T. Lappalainen, M. Calvo, G. Getz, E.T. Dermitzakis, K.G. Ardlie, R. Guigó, Human genomics. The human transcriptome across tissues and individuals, Science. 348 (2015) 660-665.

[25] N.M. Ferraro, B.J. Strober, J. Einson, N.S. Abell, F. Aguet, A.N. Barbeira, M. Brandt, M. Bucan, S.E. Castel, J.R. Davis, E. Greenwald, G.T. Hess, A.T. Hilliard, R.L. Kember, B. Kotis, Y. Park, G. Peloso, S. Ramdas, A.J. Scott, C. Smail, E.K. Tsang, S.M. Zekavat, M. Ziosi, Aradhana, TOPMed Lipids Working Group, K.G. Ardlie, T.L. Assimes, M.C. Bassik, C.D. Brown, A. Correa, I. Hall, H.K. Im, X. Li, P. Natarajan, GTEx Consortium, T. Lappalainen, P. Mohammadi, S.B. Montgomery, A. Battle, Transcriptomic signatures across human tissues identify functional rare genetic variation, Science. 369 (2020). https://doi.org/10.1126/science.aaz5900.

[26] A. Frankish, M. Diekhans, A.-M. Ferreira, R. Johnson, I. Jungreis, J. Loveland, J.M. Mudge, C. Sisu, J. Wright, J. Armstrong, I. Barnes, A. Berry, A. Bignell, S. Carbonell Sala, J. Chrast, F. Cunningham, T. Di Domenico, S. Donaldson, I.T. Fiddes, C. García Girón, J.M. Gonzalez, T. Grego, M. Hardy, T. Hourlier, T. Hunt, O.G. Izuogu, J. Lagarde, F.J. Martin, L. Martínez, S. Mohanan, P. Muir, F.C.P. Navarro, A. Parker, B. Pei, F. Pozo, M. Ruffier, B.M. Schmitt, E. Stapleton, M.-M. Suner, I. Sycheva, B. Uszczynska-Ratajczak, J. Xu, A. Yates, D. Zerbino, Y. Zhang, B. Aken, J.S. Choudhary, M. Gerstein, R. Guigó, T.J.P. Hubbard, M. Kellis, B. Paten, A. Reymond, M.L. Tress, P. Flicek, GENCODE reference annotation for the human and mouse genomes, Nucleic Acids Res. 47 (2019) D766-D773.

[27] T. Derrien, R. Johnson, G. Bussotti, A. Tanzer, S. Djebali, H. Tilgner, G. Guernec, D. Martin, A. Merkel, D.G. Knowles, J. Lagarde, L. Veeravalli, X. Ruan, Y. Ruan, T. Lassmann, P. Carninci, J.B. Brown, L. Lipovich, J.M. Gonzalez, M. Thomas, C.A. Davis, R. Shiekhattar, T.R. Gingeras, T.J. Hubbard, C. Notredame, J. Harrow, R. Guigó, The GENCODE v7 catalog of human long noncoding RNAs: analysis of their gene structure, evolution, and expression, Genome Res. 22 (2012) 1775-1789.

[28] F.A. Wolf, P. Angerer, F.J. Theis, SCANPY: large-scale single-cell gene expression data analysis, Genome Biol. 19 (2018) 15.

[29] Z. Krchňáková, P.K. Thakur, M. Krausová, N. Bieberstein, N. Haberman, M. 
Müller-McNicoll, D. Staněk, Splicing of long non-coding RNAs primarily depends on polypyrimidine tract and $5^{\prime}$ splice-site sequences due to weak interactions with SR proteins, Nucleic Acids Res. 47 (2019) 911-928.

[30] N. Gil, I. Ulitsky, Production of Spliced Long Noncoding RNAs Specifies Regions with Increased Enhancer Activity, Cell Syst. 7 (2018) 537-547.e3.

[31] A. Kuhn, A. Kumar, A. Beilina, A. Dillman, M.R. Cookson, A.B. Singleton, Cell population-specific expression analysis of human cerebellum, BMC Genomics. 13 (2012) 610.

[32] E. Khrameeva, I. Kurochkin, D. Han, P. Guijarro, S. Kanton, M. Santel, Z. Qian, S. Rong, P. Mazin, M. Bulat, O. Efimova, A. Tkachev, S. Guo, C.C. Sherwood, J. Gray Camp, S. Paabo, B. Treutlein, P. Khaitovich, Single-cell-resolution transcriptome map of human, chimpanzee, bonobo, and macaque brains, bioRxiv. (2019) 764936. https://doi.org/10.1101/764936.

[33] C. Jiang, Y. Li, Z. Zhao, J. Lu, H. Chen, N. Ding, G. Wang, J. Xu, X. Li, Identifying and functionally characterizing tissue-specific and ubiquitously expressed human IncRNAs, Oncotarget. 7 (2016) 7120-7133.

[34] R.M. Beiter, A. Fernández-Castañeda, C. Rivet-Noor, A. Merchak, R. Bai, E. Slogar, S.M. Seki, D.A. Rosen, C.C. Overall, A. Gaultier, Evidence for oligodendrocyte progenitor cell heterogeneity in the adult mouse brain, bioRxiv. (2020) 2020.03.06.981373. https://doi.org/10.1101/2020.03.06.981373.

[35] A.D. Rouillard, G.W. Gundersen, N.F. Fernandez, Z. Wang, C.D. Monteiro, M.G. McDermott, A. Ma'ayan, The harmonizome: a collection of processed datasets gathered to serve and mine knowledge about genes and proteins, Database . 2016 (2016). https://doi.org/10.1093/database/baw100.

[36] M.S. Almén, E.K. Nilsson, J.A. Jacobsson, I. Kalnina, J. Klovins, R. Fredriksson, H.B. Schiöth, Genome-wide analysis reveals DNA methylation markers that vary with both age and obesity, Gene. 548 (2014) 61-67. 